\title{
Robust Covariance Estimation for Data Fusion From Multiple Sensors
}

\author{
João Sequeira, Antonios Tsourdos, Member, IEEE, and Samuel B. Lazarus
}

\begin{abstract}
This paper addresses the robust estimation of a covariance matrix to express uncertainty when fusing information from multiple sensors. This is a problem of interest in multiple domains and applications, namely, in robotics. This paper discusses the use of estimators using explicit measurements from the sensors involved versus estimators using only covariance estimates from the sensor models and navigation systems. Covariance intersection and a class of orthogonal Gnanadesikan-Kettenring estimators are compared using the 2-norm of the estimates. A Monte Carlo simulation of a typical mapping experiment leads to conclude that covariance estimation systems with a hybrid of the two estimators may yield the best results.
\end{abstract}

Index Terms-Covariance estimation, covariance intersection (CI), data fusion, robust estimation.

\section{INTRODUCTION}

D ATA FUSION is traditionally used to increase the accuracy of the measurement being performed and to overcome unreliability in sensors or uncertainty in sensor outputs. There is another benefit of data fusion which is particularly useful for autonomous mobile robots [18], [22]. Different sources undergoing fusion are usually based on different assumptions, some of which may be invalid at any given time. By performing data fusion, the assumptions are in a way "factored out." Hence, fusion can reduce a system's dependence on invalid a priori assumptions and make the system more robust. Given that data fusion is a beneficial approach, the primary issue is how to combine or fuse the outputs of systems that are possibly disparate [3].

The literature on robust estimation of the uncertainty in a set of measurements spans a wide range of scientific disciplines, ranging from chemistry to economics and medicine, and provides a large number of estimation techniques for the respective covariance matrix. $M$-estimators linearly combine measurement vectors according to their Mahalanobis distance

Manuscript received June 16, 2010; revised December 3, 2010; accepted January 27, 2011. This work was supported in part by Fundação para a Ciência e a Tecnologia (Institute for Systems and Robotics/Instituto Superior Técnico plurianual funding) through the POS_Conhecimento Program that includes FEDER funds. The Associate Editor coordinating the review process for this paper was Dr. John Sheppard.

J. Sequeira is with the Institute for Systems and Robotics, Instituto Superior Técnico, Universidade Técnica de Lisboa, 1049-001 Lisbon, Portugal (e-mail: jseq@isr.ist.utl.pt).

A. Tsourdos and S. B. Lazarus are with the Autonomous Systems Group, Department of Informatics and Systems Engineering, Cranfield University at the U.K. Defence Academy, SN6 8LA Shrivenham, U.K. (e-mail: a.tsourdos@ cranfield.ac.uk; s.b.lazarus@ cranfield.ac.uk).

Color versions of one or more of the figures in this paper are available online at http://ieeexplore.ieee.org.

Digital Object Identifier 10.1109/TIM.2011.2141230 (see, for instance, [4]). S-estimators minimize the volume of the ellipsoid associated with a covariance matrix subject to a function of the Mahalanobis distance of the measurement samples (see, for instance, [2]). The nearest neighbor variance estimation (NNVE) [15] classifies measurements as outliers according to their distance to the cluster of $K$-nearest neighbors, with outliers showing larger distances. By estimating the distribution of the distances of nonoutliers, maximum likelihood (ML) estimators for mean and covariance can be obtained. The NNVE is argued to perform well in situations where the number of outliers is large, as in estimating minefields from noisy images.

In robotics, the navigation procedure often produces measurements that already have an associated covariance matrix, and hence, the estimation relies on the direct combination of covariance matrices. Multiple techniques for information fusion have been in use for many years in robotics, ranging from the deterministic extended Kalman filter (EKF) to the stochastic approaches using particle filters (see, for instance, [13], [14], [20], and [21]). EKF-based estimation, widely used in selflocalization methods, explicitly yields a covariance matrix. In its basis form, particle filters, also widely used for localization and mapping, do not directly yield such matrices but require its estimation from the particles at each step. The output of the fusion process is a covariance matrix expressing the uncertainty in the estimation error along the different degrees of freedom.

In multiple robot systems, if complete communication between the robots is assumed together with a priori knowledge on their respective dynamics, then typical EKF-based fusion yields a covariance estimate for each measurement obtained by a robot. However, it is seldom the case that an agent knows enough information on its teammates that can be used for EKFbased estimation, hence the need for the use of alternative strategies such as covariance intersection (CI) or even ML estimation directly from the data.

As illustrated ahead, data fusion using ML estimation tends to be conservative, meaning that it looks only for the data, implicitly assuming that it is all good, trying to minimize a quadratic error. ML works only on measurements and does not use existing covariance information that, in a sense, embeds the way that data were generated, e.g., by an EKF-based navigation system. If the data sets include measurements that do not fit the true normal distribution, i.e., they can qualify as outliers, then the resulting covariance estimate may be of poor quality.

In critical applications such as search and rescue, information from the structure of the covariance matrices associated with 
TABLE I

OGK Covariance Estimator ( $\Omega$ Is the Class Parameter)

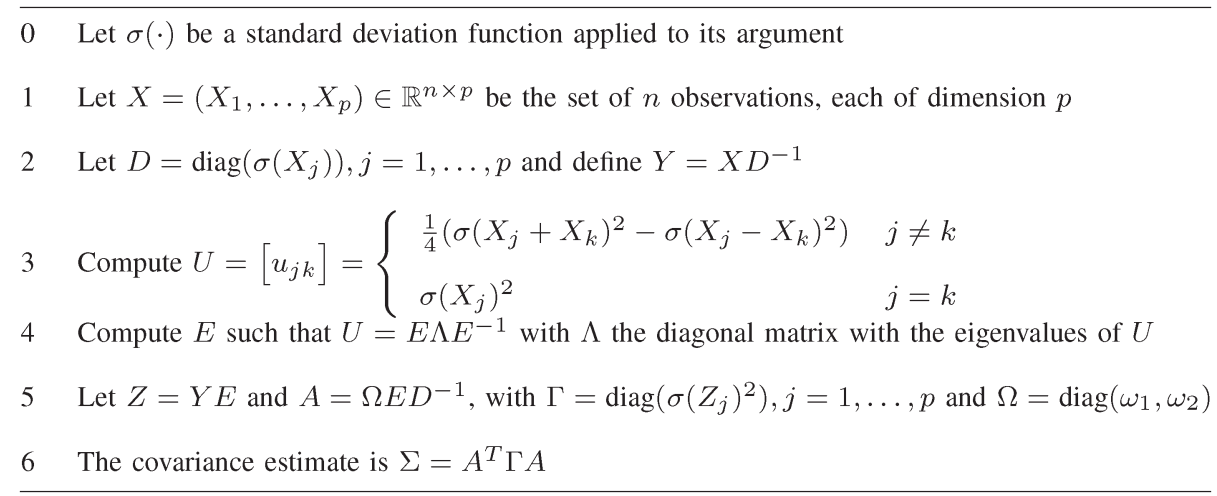

landmarks can be used to improve path planning, for instance, by having robots moving according to directions where uncertainty is maximal such that they can improve the mapping of the area. Thus, both the covariance estimates by each source and the fusion process must be robust to the presence of outlier measurements.

CI [1], [11] addresses this problem by merging the different covariances using a convex combination of the estimates. Although producing better estimates than ML estimation (in a sense to be detailed ahead), the fact that the correlation between the different information sources is not considered may decrease the quality of the estimates. Also, when the covariance of one source is "corrupted" due to outlier measurements, the resulting combination may yield poor results.

This paper focuses on the estimation of a covariance matrix for a single landmark that has been observed by two different sensors (although the framework is easily expandable to a bigger number of sensors and landmarks). The estimation of the covariance matrix is made using an orthogonal Gnanadesikan-Kettenring (OGK) estimator, based on a robust estimator from [8] (mean estimates are also important and can be provided by the OGK procedure but are not addressed in this paper). The distinctive feature of the OGK estimation is that it combines the use of the actual measurements with an existing estimate of the covariance and hence implicitly accounts for any correlation between the sources of the measurements and for the way that these were obtained. There are close relations between this type of estimation and principal component analysis in the sense that the input data set that suffers is passed through an orthogonal linear transformation.

The organization of this paper is as follows. Section II describes the OGK estimator and presents a simple example that illustrates the distinctive outlier rejection feature of this estimator. A qualitative comparison between ML, CI, and OGK estimates is provided in Section III. Section IV discusses typical quality indexes, from a robotics perspective, for covariance estimators. Section V discusses the proposed OGK-based estimator from the standpoint of statistical consistency. A hybrid strategy using both OGK and CI estimators is presented in Section VI, together with the results on several Monte Carlo experiments that motivate the final conclusions in Section VII.

\section{Robust Estimation OF A COVARIANCE MATRIX IN DATA FUSION}

When fusing data from different sensors, one can assume that the statistical measures associated with each of them are the best possible in some sense, e.g., the covariance estimated by an EKF. The presence of disturbances in sensor measurements may corrupt the estimates, although they are still optimal from the filter point of view and the filter embeds its own strategy to provide robust covariance estimates.

Sensors generating correlated data may also bias the estimates in the sense that the fusion results may be overconfident. Hence, it is interesting to test strategies that simultaneously account for the confidence of each of the sensors but also keep the conservative perspective that the sensors may be induced in error and generate outlier measurements. As a sort of generic argument, it is possible to construct hybrid estimators that take advantage of the best characteristics of more specialized estimators.

CI has been used to fuse simultaneous localization and mapping estimates, and it is shown that it is an optimal method when the cross correlation between the measurements being fused is unknown [12]. It has been reported that the correlation between the initial estimation errors may lead to a CI estimate that is too optimistic [6].

In some applications, measurements from different sensors may be correlated, e.g., as when a swarm of robot exchanges information on their relative position. Since the CI does not use any estimates of the cross correlation between the measurements by different robots may lead to overoptimistic estimates. A natural strategy to tackle this issue relies on estimating the covariance of the data from multiple sources using the measurements themselves and hence analyzing the data as a whole.

Fusing data from different sensors, which may be geographically distant, requires some sort of communication link, and hence, the amount of information to be exchanged is a relevant parameter. Often, the amount of data needed for robust covariance estimation is small and copes with bandwidth constraints in the communication link.

The class of OGK estimators used in this paper uses both actual measurements and covariance estimates and is described in Table I. It is a variation of that described in [5] and uses an estimation of the covariance directly from the measurements 


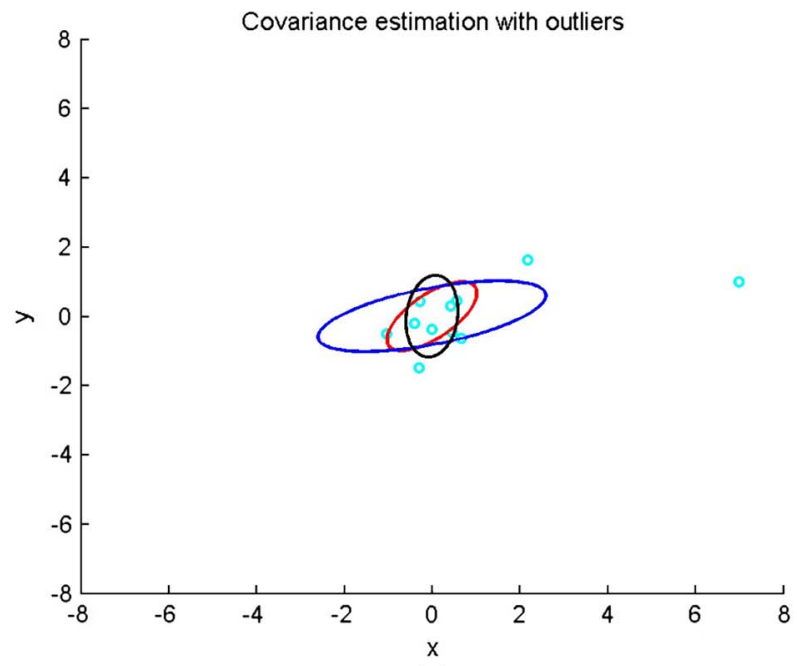

(a)

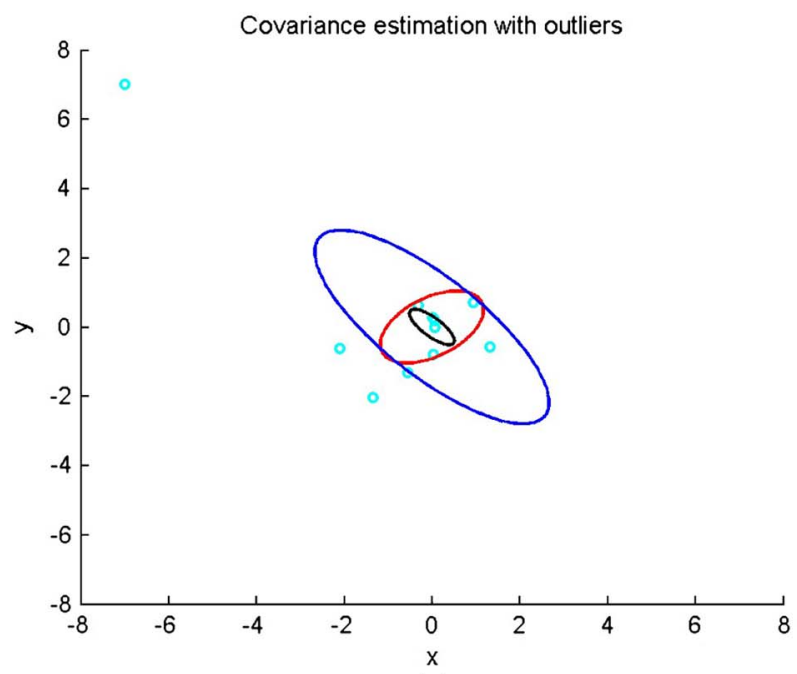

(c)

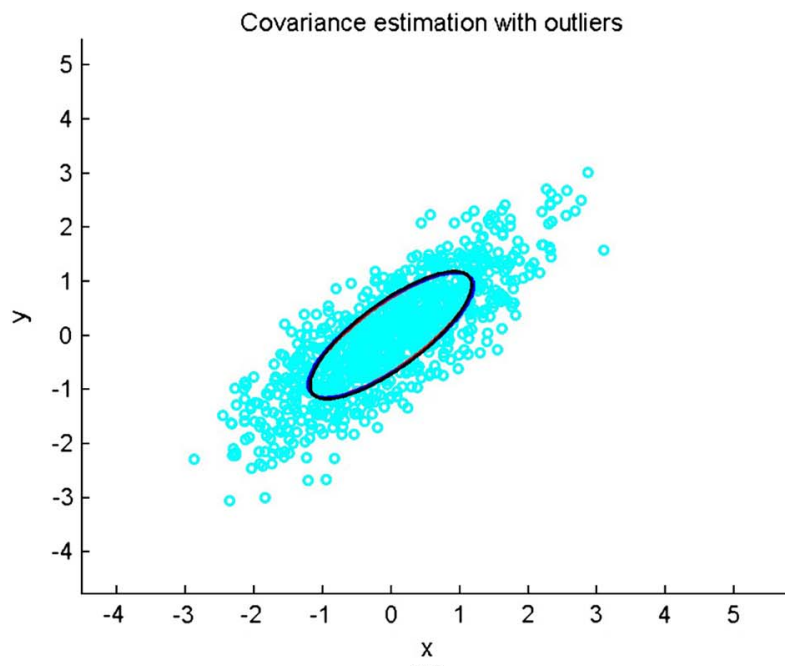

(b)

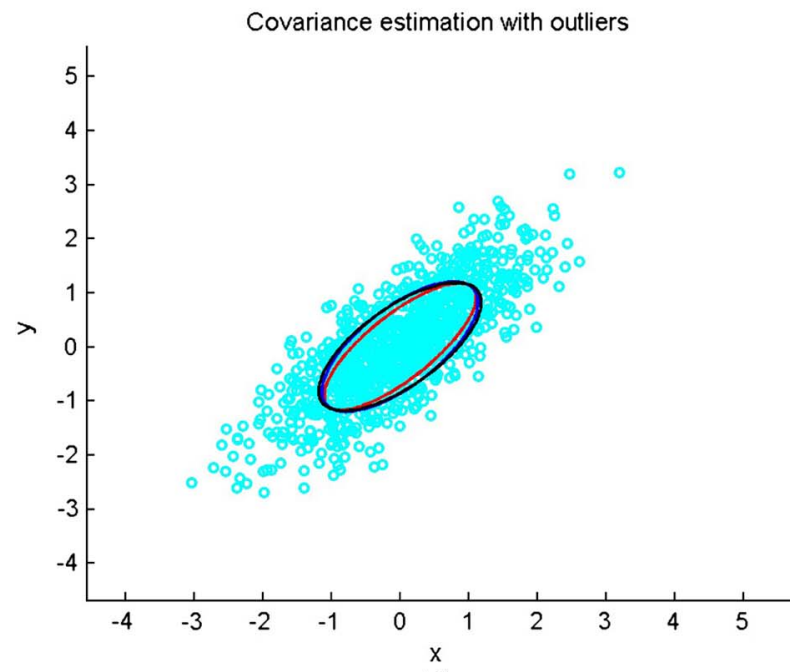

(d)

Fig. 1. Samples representing the typical behavior of the OGK estimator class for $\Omega=\mathbb{I}_{2}$. (a) Data set size 11. (b) Data set size 1001. (c) Data set size 11. (d) Data set size 1001 .

(the Gnanadesikan-Kettenring estimator), which can however be replaced by other estimates. For the purpose of the experiments, the class parameter is set to $\Omega=\mathbb{I}_{2}$.

The OGK estimator starts by scaling the input data (step 2) and computing an initial covariance estimate using the Gnanadesikan-Kettenring estimator (step 3). This initial estimate is used to obtain a new basis (step 4), formed with the eigenvectors, where the scaled data are projected and new variances are computed along each of the axis of the new frame (step 5). The data are then reverted back to the original frame (step 6). The underlying idea of the process is to extract variances accounting for the distances between the measurements. If one of the axes is much bigger than the other and the variances along this axis are small, then it is likely that the measurements that induce the length of the bigger axis are outliers. The parameters $\Omega$ allow the scaling of the covariance found.

For the purpose of this paper, $p=2$, corresponding to having the sensors operating in a plane. Fig. 1 shows a typical behavior of the estimator in Table I. The data follow a normal distribution with $(0,0)$ mean and covariance $[1,0.8 ; 0.8,1]$. The points $(7,1)$ and $(-7,7)$ are outliers generated out of the normal distribution for the upper and lower plots, respectively. The plots show the data as $\circ$ marks. The error ellipses in red and blue correspond to the ML estimates for the data without and with the outlier, respectively. The ellipse in black is the OGK estimate, also including the outlier. All the ellipses correspond to a 0.5 confidence degree.

The influence of the outlier in the estimation is clearly shown in Fig. 1(a). The common ML estimator fails completely to reject its influence. Instead, the OGK estimation is robust to the outlier, although not completely rejecting it, as can be inferred from the decrease in the error ellipse eccentricity relative to the ML estimate for the undisturbed data. The dimension of the data set induces a filtering effect that hides the influence of the outlier (see Fig. 1). If the outlier is completely misaligned with the true data, as in Fig. 1(c) and (d), then ML simply increases the uncertainty to cover the outlier as if it were true data. Instead, the OGK preserves the information on the relative position of the outlier (note the orientation of the error ellipse) but simultaneously preserves the information on the true data, maintaining the area of the error ellipse close to that of the MLE for the true data set. 


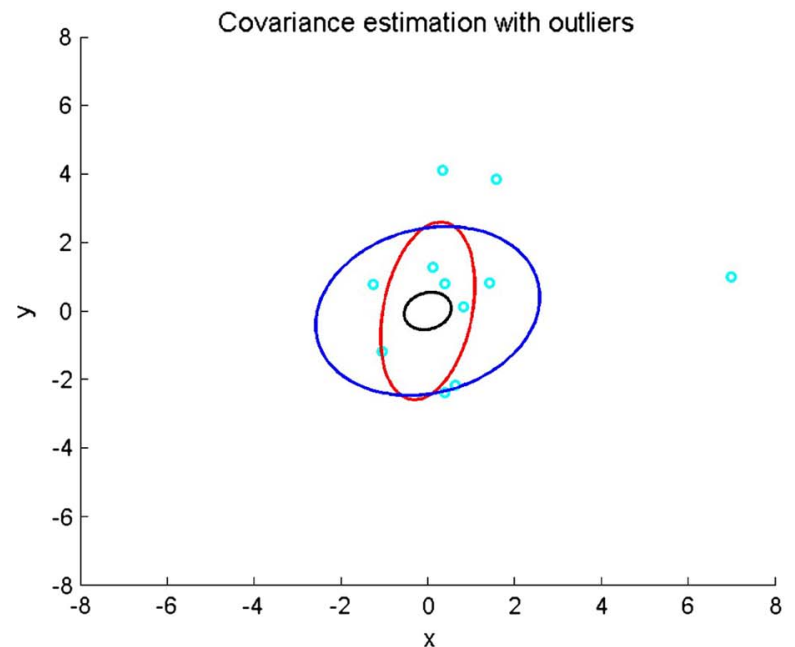

(a)

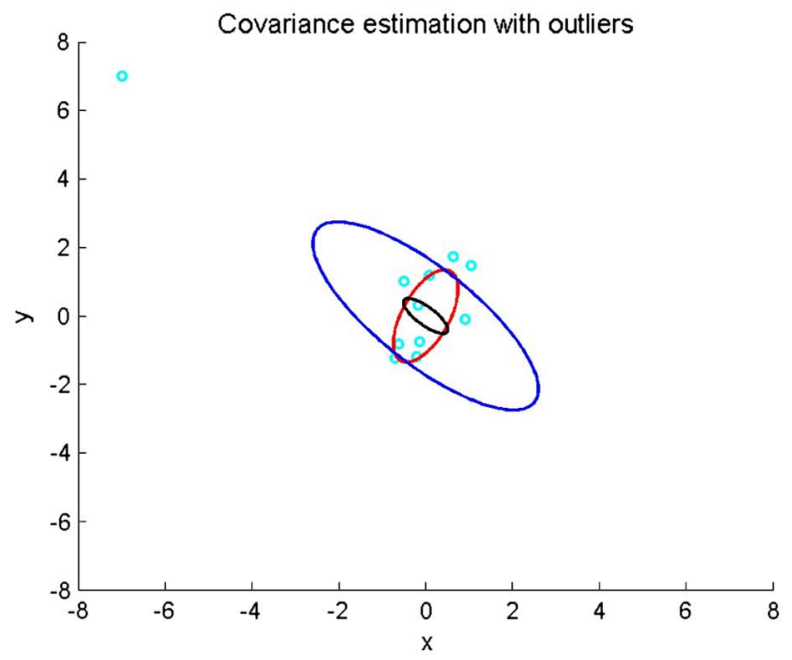

(c)

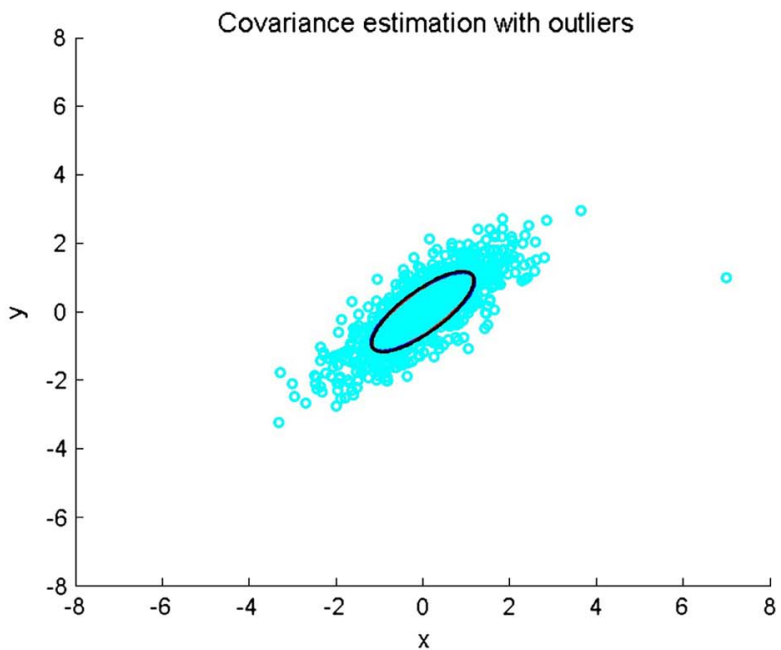

(b)

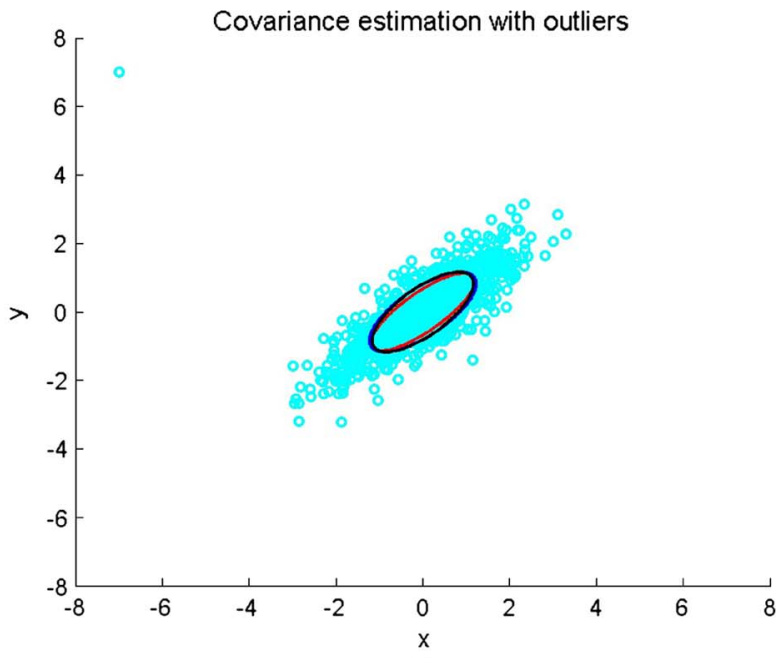

(d)

Fig. 2. Samples representing the typical behavior of the OGK estimator class for $\Omega=\mathbb{I}_{2}$. (a) Data set size 11. (b) Data set size 1001. (c) Data set size 11. (d) Data set size 1001 .

The aforementioned example illustrates an interesting feature of the OGK, namely, its ability to filter out the variances related to measurements that differ much from those of the rest of a data set.

The samples in Fig. 2 were obtained with the data set generated according a covariance $[1,0.8 ; 0.8,3]$. The sample with the smallest data set clearly shows the negative effect of the outlier. As before, when the dimension of the data set increases, the effect of the outlier vanishes in the ML estimate. The OGK estimate is also influenced by the filtering effect induced by the size of the data set, but the result is far more optimistic than that of MLE.

The structure of the algorithm clearly indicates a scaling in the input data. Moreover, the dimension of the data set and the number of outlier measurements also have decisive influence on the estimate. The outlier rejection property of the OGK estimator is obtained partially through the filtering and scaling of the variances of the input data. For example, as the dimension of the axis of greatest variance of the true data set increases, outliers aligned with that axis will tend to be considered as valid data.
Classifying a measurement as outlier means that the probability of its occurrence does not fit the known distribution for the data or that it disturbs, above some limit, the distribution being estimated for the data set.

The parameter $\Omega$ allows additional tuning of the final covariance such that a consistency criterion (defined ahead) can be met.

\section{Data Fusion Using Robust COVARIANCE ESTIMATION}

This section presents a qualitative comparison analysis on the CI and OGK estimators using a number of simulations in controlled conditions. The aim is to provide the reader with an insight on the comparison behavior of the estimators in a number of typical situations.

Two data sources are considered, each generating a 2-D random variable specifying the measurement of the position of some sensed landmark. In the first set of tests, the measurements comply to normal distributions, without outliers, i.e., no other noise is added to the measurements. The measurements of both sensors are assumed to have the same mean $(0,0)$. 


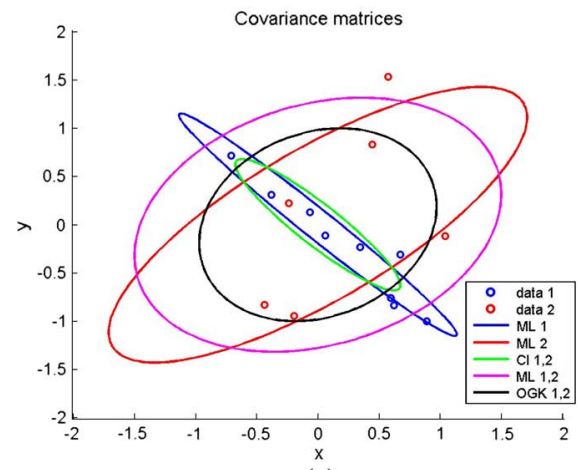

(a)

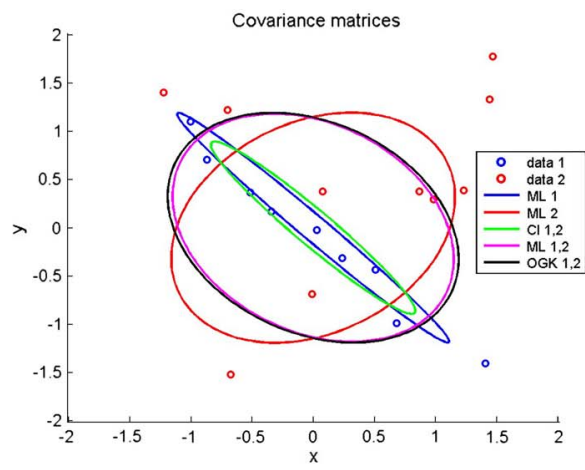

(b)

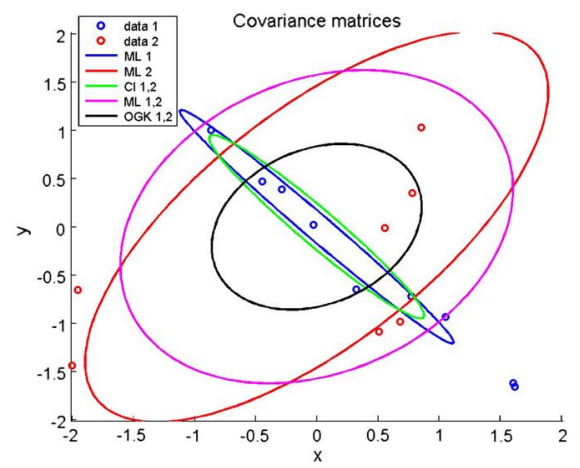

(c)

Fig. 3. Test samples for data sources that are mostly uncorrelated. (a) Sample 1. (b) Sample 2. (c) Sample 3.

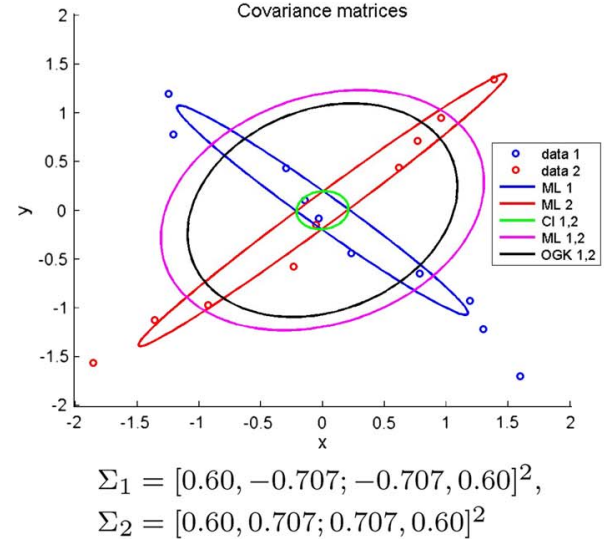

(a)

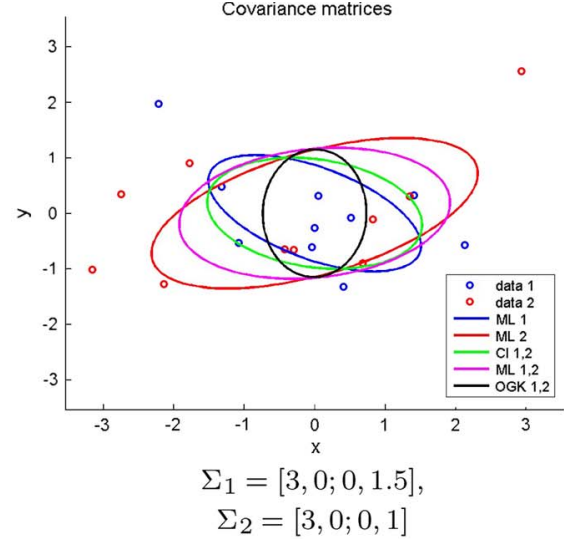

(b)

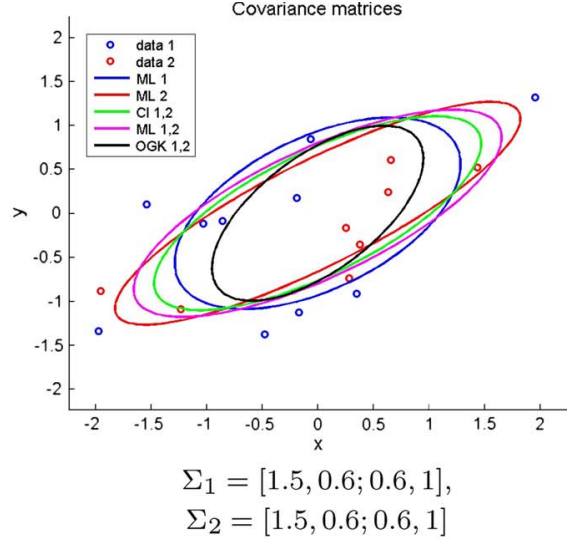

(c)

Fig. 4. Additional test samples for data sources with different correlation degrees. (a) Sample 4. (b) Sample 5. (c) Sample 6.

Fig. 3 shows three samples of the covariance estimate for the fused data obtained using CI, ML estimator, and OGK estimator. The covariances associated to the sensors are

$$
\Sigma_{1}=\left[\begin{array}{cc}
0.6 & -0.707 \\
-0.707 & 0.6
\end{array}\right]^{2} \quad \Sigma_{2}=\left[\begin{array}{cc}
0.3 & 1.5 \\
1.5 & 1
\end{array}\right]^{2}
$$

$\Sigma_{1}$ can be interpreted as coming from a highly accurate sensor along the measurement direction but with a wide detection aperture. $\Sigma_{2}$ can be identified with a sensor with poor accuracy. The preferred directions of the two sensors are almost orthogonal, meaning that the correlation between the measurements from the two sensors tends to be small.

The measurements from the two sensors are represented by the $\circ$ symbols. For the sake of comparison, all ellipses shown correspond to the same confidence degree of 0.5 . The CI estimates use a parameter value of $\omega=0.5$, which, in a sense, means that there is no preferred data source.

If the two sensors are highly uncorrelated and with the very different variances along the principal directions, then CI tends to "lock" on the sensor with the smallest variance along the principal direction. This is a reasonable behavior from the point of view of determining uniquely the best measurement direction. However, it is not necessarily the case if, for instance, the covariance information is used to plan a path that has to optimize some combination of the path length and the total uncertainty at each target. Instead, the behavior of the OGK suggests a much less optimistic estimate for the uncertainty resulting from the fusion of the data. In some occasions, this seemingly less optimistic view may still be the most reasonable, namely, when the uncertainty along the main axis is high (as in Fig. 3 sample 3).

Fig. 4 shows some additional situations, where both sensors have clearly preferred measurement directions, i.e., the eccentricity of the corresponding error ellipses is high. Also, each of the three samples corresponds to a different degree of correlation between the sources.

Sample 4 illustrates an extreme situation when sensors with close characteristics (the eigenvalues of both the covariance matrices are identical) provide measurements obtained along orthogonal directions. In what concerns the area of the error ellipses, the CI clearly outperforms the OGK. The CI returns the intersection between the two ellipses, corresponding to the region where both sensors generate data. This is a known feature of the CI method [12]. The OGK instead enlarges the corresponding error ellipse in order to cover for the whole data. Both data sets are well structured, including only measurements fitting the Gaussian distribution. This means that the OGK assumes that the large variations between them are not outliers, and hence, the corresponding covariance must be large enough to accommodate them all.

In sample 5, the characteristics of the two sensors are much closer than before, meaning that the eigenvalues of one covariance matrix are close to the corresponding ones of the 

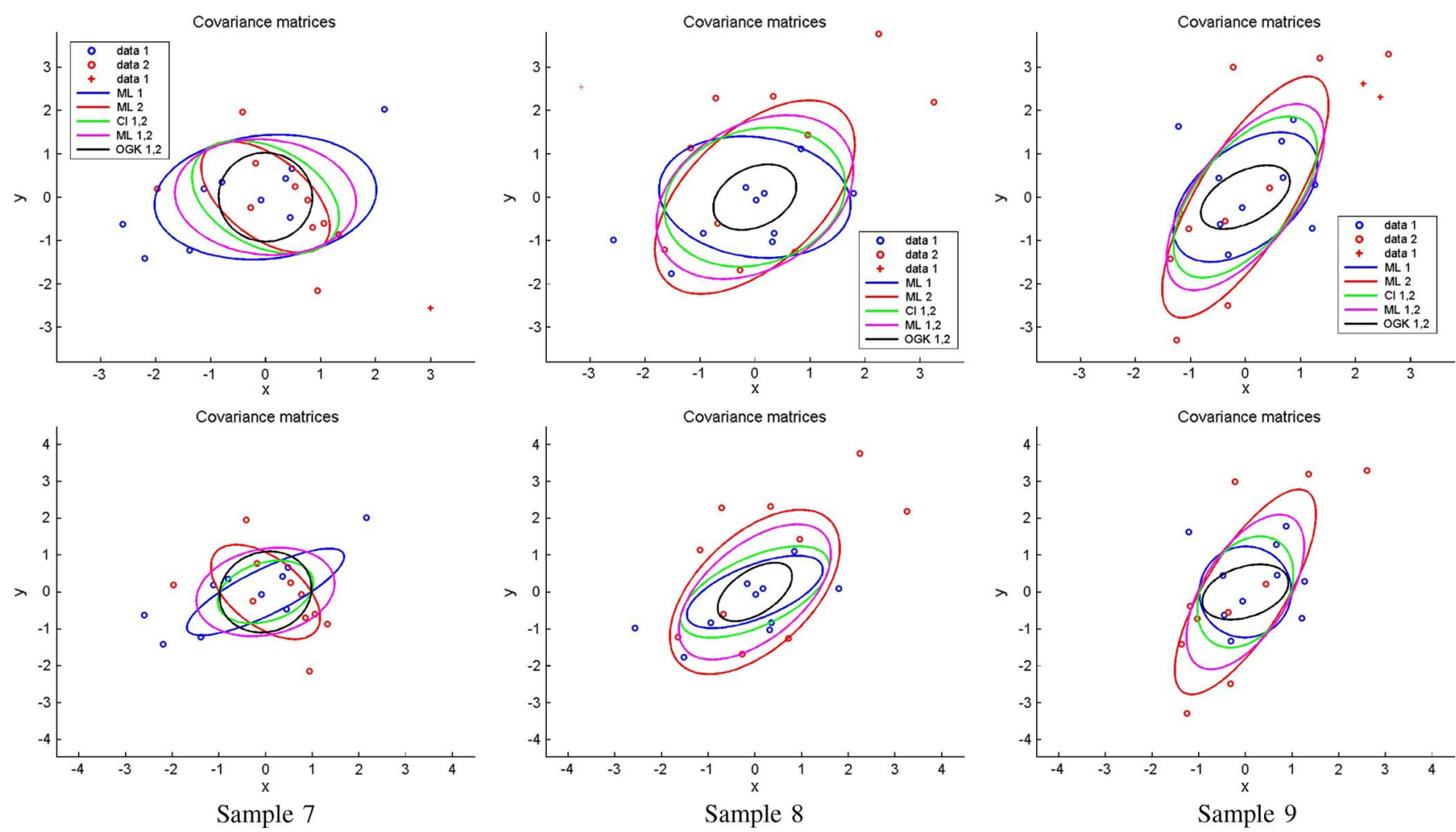

Fig. 5. Test samples for correlated data sources with additional data in data set 1.

other matrix and the corresponding eigenvectors are far from being orthogonal (as in sample 4). The CI tends to exhibit a characteristic between those of the individual sensors, whereas the OGK seems to focus on the variances along the principal directions of the whole set of data.

In the extreme situation when the two covariances are similar (sample 6), the CI estimator (with $\omega=0.5$ ) naturally yields a coherent result, as it preserves the information already in the two input covariance estimates. The OGK estimate exhibits principal directions consistent with the input data but with a slight decrease in the area of the corresponding error ellipse. The OGK behavior is clearly more optimistic (in the sense that the total uncertainty is smaller) than that of the CI.

Fig. 5 shows another set of comparative results when additional outlier measurements, marked as + , are added to the first data set. The covariances of the sample populations are $\Sigma_{1}=$ $[1.5,0.6 ; 0.6,1]$ and $\Sigma_{2}=1.5 *[1.5,0.6,0.6,1]^{-1}$ for sample 7. Samples 8 and 9 were obtained with $\Sigma_{2}=[0.3,1.5 ; 1.5,1]^{2}$. The upper plots show the covariance estimates accounting for the additional data, while the covariances in the lower plots do not include them.

As in the previous examples, the CI estimator operates on the ML estimates, and hence, it is naturally led to an erroneous estimate due to the incorrect covariance for source 1. The OGK uses the raw measurements and hence tends to outperform the $\mathrm{CI}$ in the sense that it is far less sensitive to the disturbance data.

Sample 7 clearly shows the influence of the additional data on the ML estimates. Without the additional data, both estimators show comparable performances. If the new data are seen as outliers, then, from the upper plot, the OGK estimator still maintains an eccentricity compatible with the remaining of the data. Otherwise, if the outlier is considered as coming from the real distribution, then the $\mathrm{CI}$ tends to yield an optimistic estimate, as the eccentricity is clearly emphasized relative to that in the lower plot.

In sample 8, the OGK clearly adopts an optimistic estimate (in terms of the area in the interior of the error ellipse) when compared with the CI and ML. Without the additional data, both estimates are similar and more optimistic than the MLE.

Sample 9 shows an interesting double characteristic of the OGK. If the additional data are seen as an outlier, then the OGK estimate is barely influenced. However, the decrease in the eccentricity of the ellipse shows that the data were accounted for. The CI estimate shows some robustness to the presence of the outlier data, although its influence is clearly bigger than in the OGK.

The qualitative analysis in this section is implicitly based on the empirical comparison of the eccentricities of error ellipses and on the corresponding areas covered. It is worth to remark that the scaling of the input data in the Algorithm in Table I may bias the comparison.

\section{Performance Indexes}

This section goes over the quantitative comparison between the CI and OGK estimators from a mission standpoint.

A covariance matrix $\Sigma$ expresses the average number of measurements that fall inside the associated error ellipse for some confidence degree (it is well known that the $\mathrm{RV} q^{\mathrm{T}} \Sigma^{-1} q$ has a $\chi^{2}$ distribution). The bigger the $\chi^{2}$ value is, the poorer the fitting of the data to the ellipse (it is implicitly assumed that the data do not contain any outliers). This means that, the lower a 
This article has been accepted for inclusion in a future issue of this journal. Content is final as presented, with the exception of pagination.

TABLE II

QuAlity MEASURES FOR THE Estimator TESTS

\begin{tabular}{|c|c|c|c|c|c|c|c|c|c|}
\hline \multirow[t]{3}{*}{ Measure } & \multicolumn{3}{|c|}{ Estimator } & \multicolumn{3}{|c|}{ Estimator } & \multicolumn{3}{|c|}{ Estimator } \\
\hline & CI & ML & OGK & CI & ML & OGK & CI & ML & OGK \\
\hline & \multicolumn{3}{|c|}{ Sample 1} & \multicolumn{3}{|c|}{ Sample 2} & \multicolumn{3}{|c|}{ Sample 3} \\
\hline $\operatorname{det}(\Sigma)$ & 0.01662 & 1.8989 & 0.47317 & 0.020194 & 0.88172 & 0.96733 & 0.023371 & 3.3119 & 0.26721 \\
\hline$\|\Sigma\|_{1}^{\prime}$ & 0.63591 & 1.9628 & 0.853 & 1.0913 & 1.2741 & 1.3042 & 1.2276 & 2.3523 & 0.66252 \\
\hline$\|\Sigma\|_{2}$ & 0.63377 & 1.8238 & 0.83428 & 1.057 & 1.2497 & 1.304 & 1.1833 & 2.3339 & 0.66251 \\
\hline$\|\Sigma\|_{\text {fro }}$ & 0.63432 & 2.1001 & 1.0088 & 1.0571 & 1.4351 & 1.5002 & 1.1834 & 2.7314 & 0.77563 \\
\hline$\nu(\Sigma)$ & 0.20342 & 0.75556 & 0.82452 & 0.13445 & 0.7514 & 0.75426 & 0.1292 & 0.77975 & 0.78024 \\
\hline & \multicolumn{3}{|c|}{ Sample 4} & \multicolumn{3}{|c|}{ Sample 5} & \multicolumn{3}{|c|}{ Sample 6} \\
\hline $\operatorname{det}(\Sigma)$ & 0.00084848 & 1.2655 & 0.70795 & 1.1503 & 2.5885 & 0.37467 & 0.65889 & 0.90968 & 0.28117 \\
\hline$\|\Sigma\|_{1}$ & 0.033831 & 1.5009 & 1.0563 & 1.8963 & 2.9396 & 0.96324 & 2.4015 & 3.0077 & 1.1434 \\
\hline$\|\Sigma\|_{2}$ & 0.032505 & 1.44 & 1.0532 & 1.7228 & 2.7114 & 0.9537 & 2.1276 & 2.6309 & 1.1121 \\
\hline$\|\Sigma\|_{\text {fro }}$ & 0.041689 & 1.687 & 1.2494 & 1.8477 & 2.8745 & 1.0314 & 2.15 & 2.6535 & 1.1405 \\
\hline$\nu(\Sigma)$ & 0.89612 & 0.78118 & 0.79888 & 0.62252 & 0.59338 & 0.64182 & 0.38153 & 0.36253 & 0.47679 \\
\hline
\end{tabular}

norm of $\Sigma$ is, the bigger the confidence that the data fit to the estimated covariance, and hence, norms related to the area of the ellipse are acceptable quality indexes. ${ }^{1}$

Minimizing the area under the estimation error ellipse is a common goal (as in CI-based fusion; see [12]). In addition, as aforementioned, eccentricity also provides useful information on the quality of the estimation. Eccentric ellipses indicate preferred directions for further exploration of the nearby space. However, as can be seen in some of the aforementioned examples, it may also be interesting to lose eccentricity and favor a smaller area. Therefore, a combination of the two measures may prove interesting.

The $\operatorname{det}(\Sigma)$ measure is common in CI context, and it is closely related to the Frobenius norm $\sqrt{\operatorname{sum}\left(\operatorname{diag}\left(\Sigma^{\mathrm{T}} \Sigma\right)\right)}$. $\|\Sigma\|_{2}$ is the largest singular value of the matrix. To include information on the eccentricity of a covariance ellipsoid, one can propose the measure

$$
\nu(\Sigma)=\sqrt{\min (\operatorname{eig}(\Sigma)) / \max (\operatorname{eig}(\Sigma))}
$$

which is, in fact, the inverse of the so-called condition number for the ellipsoid. ${ }^{2}$

For the 2-D case, the area of the error ellipse corresponding to the $\Sigma$ covariance is $A(\Sigma)=\pi \lambda_{1}(\Sigma) \lambda_{2}(\Sigma)$, and hence, the relation with the $\nu(\Sigma)$ measure is

$$
\sqrt{A(\Sigma)} / \nu(\Sigma)=\sqrt{\pi}\|\Sigma\|_{2} .
$$

The lower the $\|\Sigma\|_{2}$, the more interesting the estimator is.

Table II shows the values obtained from the previous tests for a number of quality measures.

The results show an empirical evidence that the values $\left\|\Sigma_{\text {OGK }}\right\|_{2}$ are consistently lower than those of $\left\|\Sigma_{\mathrm{CI}}\right\|_{2}$ when the input data sets are uncorrelated. Using, from the example, the 2-norm, samples 1, 2, and 4 are examples where the CI outperforms the OGK, whereas the opposite happens in samples 3, 5 , and 6.

The aforementioned indexes also suggest that each of the CI and OGK methods has preferred regions of operation, depend-

\footnotetext{
${ }^{1} \mathrm{~A}$ practical consequence of the theorem on the equivalence between norms in $\mathbb{R}^{n}$ is that any norm in $\mathbb{R}^{n}$ can be used.

${ }^{2}$ Matlab operators sum, diag, and eig operators are used here to simplify the notation.
}

ing on the degree of correlation between the data sets. This suggests that a hybrid of the two methods is used.

\section{CONSISTENCY OF THE Class OF OGK Estimators}

When the two data sources show a low degree of correlation, as when the axis of major (respectively minor) variances is orthogonal, the CI estimate is consistent in the sense that the deviation between the estimated covariance for the fused data $\Sigma$ and the correlation of the error between the estimated and real values of the mean obtained by the fusion $\Sigma_{\text {error }}$ is always positive semidefinite (see [11] and [19])

$$
\Sigma-\Sigma_{\text {error }} \geq 0 \text {. }
$$

The eigenvalues of $\Sigma$ may be bigger than those of $\Sigma_{\text {error }}$. Given that both $\Sigma$ and $\Sigma_{\text {error }}$ are Hermitian, using the Rayleigh-Ritz theorem [9], for any vector $x$

$$
\begin{gathered}
\lambda_{n}(\Sigma) x^{\star} x \leq \frac{x^{\star} \Sigma x}{x^{\star} x} \leq \lambda_{1}(\Sigma) x^{\star} x \\
\lambda_{n}\left(\Sigma_{\text {error }}\right) x^{\star} x \leq \frac{x^{\star} \Sigma_{\text {error }} x}{x^{\star} x} \leq \lambda_{1}\left(\Sigma_{\text {error }}\right) x^{\star} x
\end{gathered}
$$

with $\lambda_{1}(\cdot)$ and $\lambda_{n}(\cdot)$ as the biggest and smallest eigenvalues of the argument matrices, respectively, and hence

$$
\begin{aligned}
\left(\lambda_{n}(\Sigma)-\lambda_{1}\left(\Sigma_{\text {error }}\right)\right) x^{\star} x & \leq \frac{x^{\star}\left(\Sigma-\Sigma_{\text {error }}\right) x}{x^{\star} x} \\
& \leq\left(\lambda_{1}(\Sigma)-\lambda_{n}\left(\Sigma_{\text {error }}\right)\right) x^{\star} x .
\end{aligned}
$$

Given that both $\Sigma \geq 0$ and $\Sigma_{\text {error }} \geq 0$ (see [16, Th. 8.25]) and that (2) must hold for any two matrices $\Sigma, \Sigma_{\text {error }}$

$$
\begin{aligned}
\lambda_{n}(\Sigma)-\lambda_{1}\left(\Sigma_{\text {error }}\right) & \leq \lambda_{n}\left(\Sigma-\Sigma_{\text {error }}\right) \\
\lambda_{1}\left(\Sigma-\Sigma_{\text {error }}\right) & \leq \lambda_{1}(\Sigma)-\lambda_{n}\left(\Sigma_{\text {error }}\right) .
\end{aligned}
$$

Therefore, in order for (1) to hold, it is necessary that $\lambda_{1}(\Sigma) \geq \lambda_{n}\left(\Sigma_{\text {error }}\right)$, which can be interpreted as the estimate $\Sigma$ being conservative in the sense that the uncertainty expressed by $\Sigma$ is bigger than that expressed by the correlation of the estimation error $\Sigma_{\text {error }}$, at least along the direction of the eigenvector associated to $\left.\lambda_{1}(\Sigma)\right)$.

In general, $\Sigma_{\text {error }}$ is difficult to obtain as the real value of the fused mean is unknown; only the estimate is available. 


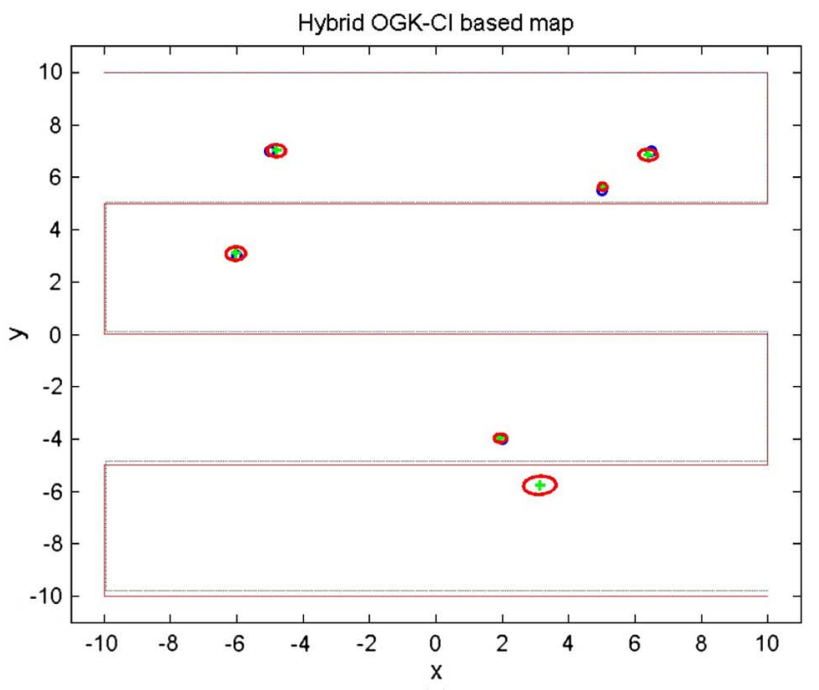

(a)

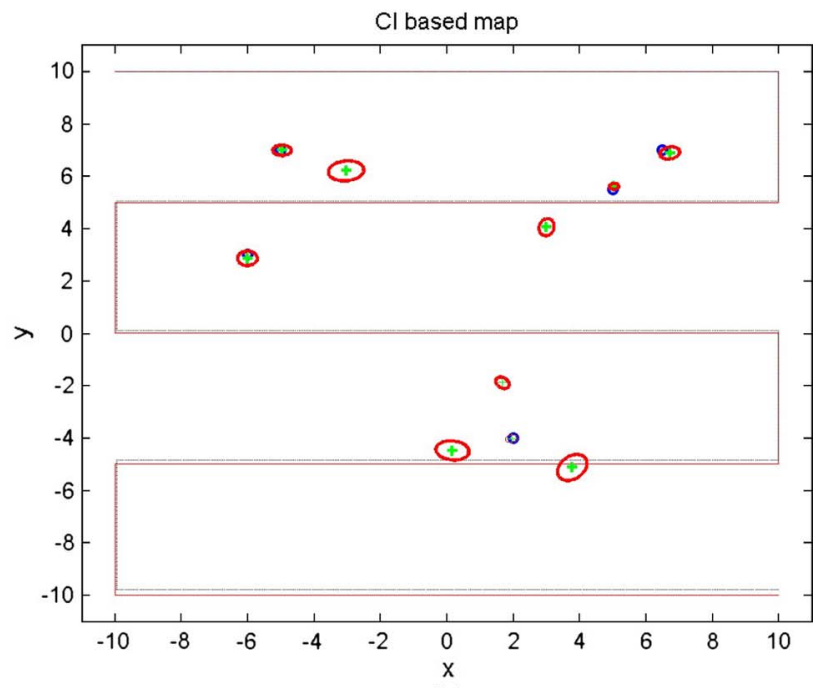

(b)

Fig. 6. Typical maps obtained for a unit power Gaussian noise and including outliers. (a) OGK-CI. (b) CI.

The OGK algorithm in [5] uses an estimate for the mean as $\mu_{\mathrm{OGK}}=A \nu$, with $A$ as the matrix defined in step 5 of Algorithm 1 and $\nu$ as a vector of means of the $Z$ variable in the algorithm. Thus, in the OGK version in [5], the parameter matrix $\Omega$ also affects the estimate $\mu_{\mathrm{OGK}}$.

However, from an application viewpoint, $\Omega$ represents a free parameter that can be used to tune the estimated covariance to the adequate conditions independently of the estimate of the fused mean. From step 5 in Algorithm 1, $\Sigma_{\mathrm{OGK}}=$ $\left(E D^{-1}\right)^{\mathrm{T}} \Omega \Gamma \Omega\left(E D^{-1}\right)$, and hence, $\Omega$ can be used to scale the eigenvalues of $\Sigma_{\text {OGK }}$ while preserving the eigenvectors. From (2), if only the matrix $\Sigma_{\text {OGK }}$ has its eigenvalues scaled, leaving $\Sigma_{\text {error }}$ unchanged, then the left-hand term can be made positive, which means that (1) holds. For this, it is enough to select $\Omega$ such that the smallest eigenvalue of $\Sigma$ is large enough. Therefore, by proper choice of $\Omega$, the class of estimators in Table I can always be made consistent in the sense of (1) and eventually optimize other criteria.

A more strict notion of consistency involves the checking of convergence to the true value of the estimator as the dimension of the sample increases. An estimator sequence is weakly consistent if it converges in probability to the true value of the estimator as the number of tests $n$ grows, i.e., it verifies Chebychev's inequality (see, for instance, [10]). The CI can be shown to be consistent in this strict sense. It is enough to consider two data sets generated by identical distributions, and it is clear that the resulting fused covariance matrix is identical to those used to generate the data sets.

Instead, a simple simulation shows that the OGK does not have identical property. In fact, generating a data set normally distributed for some arbitrary covariance matrix, the corresponding OGK covariance estimate does not converge to the original covariance no matter the size of the data set.

\section{Hybrid Sensor Fusion and CASE Study}

Both fusion strategies can be mixed in a hybrid OGK-CI system. Following the discussion in Section IV on the use 2-norm quality measure, the decision on which strategy to use is based simply on the direct comparison of the 2-norm values for each of the strategies.

At each iteration, $\Sigma_{\mathrm{CI}}$ and $\Sigma_{\mathrm{OGK}}$ are computed, and the selection is made using the rule

$$
\Sigma=\arg \min \left(\left\|\Sigma_{\mathrm{CI}}\right\|_{2},\left\|\Sigma_{\mathrm{OGK}}\right\|_{2}\right) .
$$

$\Sigma$ is thus the covariance matrix representing the uncertainty in the target position estimates at a given iteration that minimizes the 2-norm performance index.

To illustrate the performance of this hybrid fusion strategy, a mapping application is demonstrated in simulation. The environment is a planar area with five static targets. These are to be mapped by a single robot moving along a predefined path. The robot is equipped with a single range sensor that returns an estimate for the position of a target. The sensor is assumed to have a detection cone with $1^{\circ}$ aperture, and the range measurements are corrupted by Gaussian noise. The measurements are further corrupted by Gaussian outliers of fixed variance, with the time between consecutive outliers being exponentially distributed. The robot contains a local map that is updated periodically to contain the targets identified. At each instant, a measurement is taken by the sensor onboard the robot. Once a target is placed in the local map, it can be fused with others, or it is kept unchanged; it is never deleted.

The data association policy encompasses three stages and is identical for both strategies and based on grouping neighbor measurements. In the first stage, a measurement is fused with the existing map if it falls inside the error ellipse of some target already in the map. If this condition is not verified, the measurement is added to the map as a new target. At the second stage, after the end of the run, any two targets with overlapping error ellipses are fused together. The final stage groups targets lying in a small common neighborhood and selects only one of them to represent the real target. No fusion occurs at this stage. The $\Sigma_{\mathrm{CI}}$ and $\Sigma_{\mathrm{OGK}}$ are thus computed only at the first and second stages. 
TABLE III

Data From Monte CARLo EXPERIMENTS

\begin{tabular}{c|cccc}
\hline Run & Noise power/ $\sqrt{1.414}$ & $\lambda$ & Targets detected index & Covariance index \\
\hline 1 & 0.1 & 10 & 57 & 56 \\
2 & 0.1 & 100 & 76 & 100 \\
3 & 0.1 & 150 & 75 & 100 \\
4 & 0.5 & 10 & 50 & 53 \\
5 & 0.5 & 100 & 78 & 60 \\
6 & 0.5 & 150 & 85 & 54 \\
7 & 1 & 10 & 51 & 44 \\
8 & 1 & 100 & 77 & 47 \\
9 & 1 & 150 & 84 & 57 \\
\hline
\end{tabular}

A measurement detecting a target has associated a position and a covariance matrix. In what concerns the CI, these data are fused with the data of each of the targets already on the local map that have been associated with this one. The OGK estimate is computed by first joining the target position estimate corresponding to the measurement to the complete set of measurements taken by the robot along the path that were associated to this target and applying Algorithm 1 afterward.

Fig. 6 shows the typical runs when CI and a hybrid OGK-CI fusion are used. The robot moves along straight lines such that the sensor range covers the whole area. A unit power Gaussian noise was used. During the mission in the left-hand plot, the OGK estimator was called a total of 30 times. A total of four outliers was generated during this run. For the CI-only map, in the right-hand plot, a total of five outliers was generated. The plots suggest that the hybrid estimator has a better performance than the CI alone. Even with the additional outlier, the total number of false targets detected by the $\mathrm{CI}$ alone is clearly bigger than when using the hybrid, and these are scattered on the whole area.

Monte Carlo experiments have been performed to compare the two strategies. Each experiment involved 100 runs and different conditions for measurement noise and outliers. The robot is set to travel at $0.5 \mathrm{~m} / \mathrm{s}$, and the range measurements are taken at $10-\mathrm{Hz}$ sampling rate. The performance indexes considered indicate the number of runs in which the OGK-CI strategy outperforms the CI strategy according to two factors: 1) the number of detected targets and 2) the average 2-norm of the covariances obtained for each of the five real targets. Failing to detect all the five real targets is considered a miss. Table III shows the data for the Monte Carlo experiments and performance indexes obtained. The $\lambda$ column indicates the mean time between outliers. The "targets detected index" is the number of runs for which the number of targets detected by the OGK-CI strategy was smaller than or equal to the number detected by the $\mathrm{CI}$ alone and for which all of the real targets were detected. The "covariance index" is the number of runs for which the average $\|\Sigma\|_{2}$ value, computed over all the real targets using OGK-CI, is better than the corresponding value computed using only the CI strategy.

The results suggest that the hybrid OGK-CI strategy outperforms the CI-based strategy when the mean time between outliers $\lambda$ disturbing the sensor measurements decreases. This is verified even for moderate amounts of noise power in measurements and is an indicator of interesting filtering properties of the OGK-CI that reduce the number of false positives. The average 2-norm over the set of real targets tends to favor the OGK-CI for low measurement noise and outlier levels.

\section{CONCLUSION}

This paper has compared the CI and OGK covariance estimators for the fusion of information from two sources.

The comparison between the two estimators uses the 2norm of the estimated covariance matrix. The analysis on the relevant bounds for the two measures shows that, in worst case conditions, there are regions of the spectrum of the covariance matrix where each of the estimators outperforms the other. This suggests that a hybrid of the two estimators can provide the best results in a generic application.

Monte Carlo experiments in a mapping application show an interesting side effect, namely, that of leading to a smaller number of false positives in target detection and identification while preserving the quality of the covariances estimated for each target.

The application of the OGK estimation technique to 3$\mathrm{D}$ applications needs a recalculation of the bounds involved. Still, the same basic principles apply. Future work includes the testing of different forms of hybridizing the OGK and CI covariance estimators in the collaborative fusion of information from multiple unmanned aerial vehicles in mapping missions. More generally, robotics applications dealing with the multiple sensor fusion problem may use single of hybrid fusion strategies such as the one described in this paper. Cooperative search missions involving, for instance, ground and aerial vehicles are a natural class of applications.

A complementary research line to be explored in future work is the use of alternative covariance estimators, such as the $M$ and $S$ estimators referred in Section I, which may yield interesting results in the hybrid technique discussed in this paper.

\section{APPENDIX \\ COMPARING THE CI AND OGK ESTIMATORS}

Given the performance measures considered in the previous section, to compare the CI and OGK estimators, closed-form solutions for the eigenvalues of the estimates $\Sigma_{\mathrm{CI}}$ and $\Sigma_{\mathrm{OGK}}$ as functions of the eigenvalues of covariance matrices $A$ and $B$ are required. In both cases, getting such solutions results in algebraic lengthy expressions that are hard to compare. Eventually, good bounds on such expressions are also difficult to derive. 
This section follows [17] closely, using Weyl's inequalities (see, for instance, [7] and [9]) to compare the two estimators.

In general, unless the bounding errors are known, the comparison between bounds of performance measures for two different algorithms does not allow a direct comparison of the algorithms themselves (only of the bounds). However, they may give good indications on their expected behavior, i.e., if upper and lower bounds on the performance measures are available, where conditions can be stated under which one of the strategies performs always better than the other. In such cases, it may be worth to keep the two strategies running in parallel and choose the better one in run time.

A) Bounds on the CI Estimator: Listing the eigenvalues of a Hermitian matrix in decreasing order $\lambda_{1} \geq \lambda_{2} \geq \cdots \geq \lambda_{m}$, the eigenvalues of the sum $\Sigma^{-1}=\omega A+(1-\omega) B$ of any two Hermitian matrices verify Weyl's inequalities

$$
\lambda\left(\Sigma^{-1}\right)_{i+j-1} \leq \lambda(A)_{i}+\lambda(B)_{j}, \quad \text { for } i+j-1 \leq n
$$

where $A$ and $B$ correspond to the inverses of the covariance matrices used by the CI algorithm. Assuming that each of the sources of information is of dimension $p=2$, meaning that the robots are assumed to move on a 2-D surface, and denoting by $A$ and $B$ the inverses of the respective covariance matrices and by $\Sigma_{\mathrm{CI}}$ the CI estimate, Weyl's inequalities yield

$$
\begin{aligned}
\lambda_{1}\left(\Sigma_{\mathrm{CI}}^{-1}\right) & \leq \lambda_{1}(\omega A)+\lambda_{1}((1-\omega) B) \\
& =\omega \lambda_{1}(A)+(1-\omega) \lambda_{1}(B) \\
\lambda_{2}\left(\Sigma_{\mathrm{CI}}^{-1}\right) & \leq \lambda_{1}(\omega A)+\lambda_{2}((1-\omega) B) \\
& =\omega \lambda_{1}(A)+(1-\omega) \lambda_{2}(B) \\
\lambda_{2}\left(\Sigma_{\mathrm{CI}}^{-1}\right) & \leq \lambda_{2}(\omega A)+\lambda_{1}((1-\omega) B) \\
& =\omega \lambda_{2}(A)+(1-\omega) \lambda_{1}(B) .
\end{aligned}
$$

Using

$$
\begin{aligned}
& \lambda_{1}\left(\Sigma_{\mathrm{CI}}\right)=1 / \lambda_{2}\left(\Sigma_{\mathrm{CI}}^{-1}\right) \\
& \lambda_{2}\left(\Sigma_{\mathrm{CI}}\right)=1 / \lambda_{1}\left(\Sigma_{\mathrm{CI}}^{-1}\right)
\end{aligned}
$$

one can write the following lower bounds:

$$
\begin{aligned}
\frac{1}{\max \left\{\begin{array}{l}
\omega \lambda_{1}(A)+(1-\omega) \lambda_{2}(B), \\
\omega \lambda_{2}(A)+(1-\omega) \lambda_{1}(B)
\end{array}\right\}} & \leq \lambda_{1}\left(\Sigma_{\mathrm{CI}}\right) \\
\frac{1}{\omega \lambda_{1}(A)+(1-\omega) \lambda_{1}(B)} & \leq \lambda_{2}\left(\Sigma_{\mathrm{CI}}\right) .
\end{aligned}
$$

Any two square matrices $A$ and $B$ verify

$$
\operatorname{det}\left(\Sigma_{\mathrm{CI}}^{-1}\right) \equiv \operatorname{det}(A+B) \geq \operatorname{det}(A)+\operatorname{det}(B)
$$

and hence

$$
\lambda_{1}\left(\Sigma_{C I}^{-1}\right) \lambda_{2}\left(\Sigma^{-1}\right) \geq \omega^{2} \lambda_{1}(A) \lambda_{2}(A)+(1-\omega)^{2} \lambda_{1}(B) \lambda_{2}(B)
$$

or

$$
\lambda_{2}\left(\Sigma_{\mathrm{CI}}\right) \leq \frac{1}{\lambda_{1}(\Sigma)\left(\omega^{2} \lambda_{1}(A) \lambda_{2}(A)+(1-\omega)^{2} \lambda_{1}(B) \lambda_{2}(B)\right)}
$$

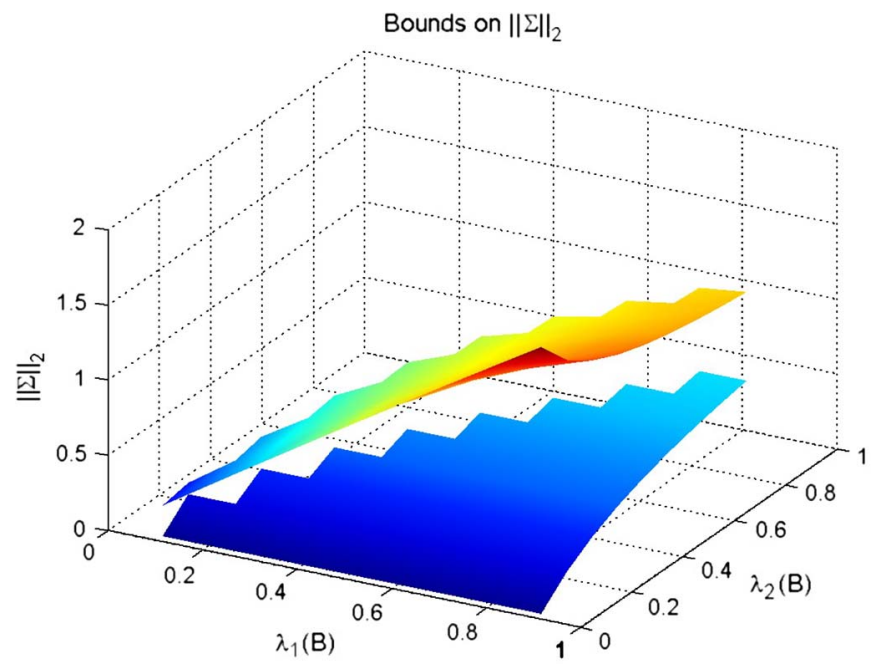

Fig. 7. Upper and lower bounds for $\left\|\Sigma_{\mathrm{CI}}\right\|_{2}$.

$$
\begin{aligned}
& \leq \frac{1}{\min \left(\lambda_{1}(\Sigma)\right)\left(\omega^{2} \lambda_{1}(A) \lambda_{2}(A)+(1-\omega)^{2} \lambda_{1}(B) \lambda_{2}(B)\right)} \\
& \leq \frac{\max \left\{\begin{array}{l}
\omega \lambda_{1}(A)+(1-\omega) \lambda_{2}(B), \\
\omega \lambda_{2}(A)+(1-\omega) \lambda_{1}(B)
\end{array}\right\}}{\omega^{2} \lambda_{1}(A) \lambda_{2}(A)+(1-\omega)^{2} \lambda_{1}(B) \lambda_{2}(B)}
\end{aligned}
$$

Similarly for $\lambda_{1}\left(\Sigma_{C I}\right)$, yielding the following upper bound:

$$
\begin{aligned}
& \lambda_{1}(\Sigma) \\
& \quad \leq \frac{1}{\lambda_{2}(\Sigma)\left(\omega^{2} \lambda_{1}(A) \lambda_{2}(A)+(1-\omega)^{2} \lambda_{1}(B) \lambda_{2}(B)\right)} \\
& \quad \leq \frac{1}{\min \left(\lambda_{2}(\Sigma)\right)\left(\omega^{2} \lambda_{1}(A) \lambda_{2}(A)+(1-\omega)^{2} \lambda_{1}(B) \lambda_{2}(B)\right)} \\
& \quad \leq \frac{\omega \lambda_{1}(A)+(1-\omega) \lambda_{1}(B)}{\omega^{2} \lambda_{1}(A) \lambda_{2}(A)+(1-\omega)^{2} \lambda_{1}(B) \lambda_{2}(B)}
\end{aligned}
$$

from which the upper bound of $\left\|\Sigma_{\mathrm{CI}}\right\|_{2}=\lambda_{1}\left(\Sigma_{\mathrm{CI}}\right)$ can be computed.

Fig. 7 shows the above bounds for a limited region of the covariance matrix spectrum. For the sake of representation, $\lambda_{1}(A)=\lambda_{2}(A)=0.5$, and $\omega=0.5$.

By construction, $\lambda_{1}(B) \geq \lambda_{2}(B)$, meaning that only this region is meaningful.

B) Bounds on the OGK Estimator: In the form in Table I, the OGK estimator does not use directly the covariance matrices associated to each of the landmarks (although, as mentioned before, step 3 can use the result of, for example, a CI estimate).

For the purpose of the OGK analysis, denote the set of $n$ measurements from the two sources $A$ and $B$ as $X=$ $\left[X_{1}, X_{2}\right]$ and $X_{1}=\left[x_{A 1}, \ldots, x_{A n}, x_{B 1}, \ldots, x_{B n}\right]^{\mathrm{T}}, X_{2}=$ $\left[y_{A 1}, \ldots, y_{A n}, y_{B 1}, \ldots, y_{B n}\right]^{\mathrm{T}}$. Measurements from sources $A$ and $B$ have covariances $A_{X}$ and $B_{X}$, respectively

$$
\begin{aligned}
& A_{X}=\left[\begin{array}{cc}
\sigma\left(X_{A_{x x}}\right)^{2} & \sigma\left(X_{A_{x y}}\right) \\
\sigma\left(X_{A_{y x}}\right) & \sigma\left(X_{A_{y y}}\right)^{2}
\end{array}\right] \\
& B_{X}=\left[\begin{array}{cc}
\sigma\left(X_{B_{x x}}\right)^{2} & \sigma\left(X_{B_{x y}}\right) \\
\sigma\left(X_{B_{y x}}\right) & \sigma\left(X_{B_{y y}}\right)^{2}
\end{array}\right] .
\end{aligned}
$$


When the measurements of source $B$ are appended to those of source $A$, the resulting covariance is

$$
C_{X}=\left[\begin{array}{cc}
\sigma\left(X_{C_{x x}}\right)^{2} & \sigma\left(X_{C_{x y}}\right) \\
\sigma\left(X_{C_{y x}}\right) & \sigma\left(X_{C_{y y}}\right)^{2}
\end{array}\right]
$$

which, assuming that both sources generate the measurements with the same mean, can be written as

$$
C_{X}=\frac{1}{2}\left[\begin{array}{cc}
\sigma\left(X_{A_{x x}}\right)^{2}+\sigma\left(X_{B_{x x}}\right)^{2} & \sigma\left(X_{A_{x y}}\right)+\sigma\left(X_{B_{x y}}\right) \\
\sigma\left(X_{A_{y x}}\right)+\sigma\left(X_{B_{y x}}\right) & \sigma\left(X_{A_{y y}}\right)^{2}+\sigma\left(X_{B_{y y}}\right)^{2}
\end{array}\right]
$$

From Table I, one can write the estimate in a generic form

$$
\begin{aligned}
\Sigma_{\mathrm{OGK}}= & \left(E D^{-1}\right)^{\mathrm{T}} \Gamma E D^{-1}=D^{-T} E^{\mathrm{T}} \Gamma E D^{-1} \\
= & D^{-1} E^{\mathrm{T}} \Gamma E D^{-1} \\
= & {\left[\begin{array}{cc}
\sigma\left(X_{C_{x x}}\right)^{-1} & 0 \\
0 & \sigma\left(X_{C_{y y}}\right)^{-1}
\end{array}\right]\left[\begin{array}{ll}
e_{11} & e_{12} \\
e_{21} & e_{22}
\end{array}\right] } \\
& \times\left[\begin{array}{cc}
\sigma\left(C_{Z_{x x}}\right)^{2} & 0 \\
0 & \sigma\left(C_{Z_{y y}}\right)^{2}
\end{array}\right] \\
& \times\left[\begin{array}{cc}
e_{11} & e_{21} \\
e_{12} & e_{22}
\end{array}\right]\left[\begin{array}{cc}
\sigma\left(X_{C_{x x}}\right)^{-1} & 0 \\
0 & \sigma\left(X_{C_{y y}}\right)^{-1}
\end{array}\right] .
\end{aligned}
$$

Also from Table I, the covariance matrix for the transformed $Z$ variable $C_{Z}$ is given by

$$
C_{Z}=E^{\mathrm{T}} D^{-\mathrm{T}} C_{X} D^{-1} E=E^{\mathrm{T}} D^{-1} C_{X} D^{-1} E
$$

from where $\sigma\left(C_{Z_{x x}}\right)^{2}$ and $\sigma\left(C_{Z_{y y}}\right)^{2}$ can be computed.

Using Schwarz inequality

$$
\left\|\Sigma_{\mathrm{OGK}}\right\| \leq\left\|D^{-1}\right\|\|E\|\|\Gamma\|\left\|E^{\mathrm{T}}\right\|\left\|D^{-1}\right\|
$$

and since $E$ can be assumed unitary without losing generality

$$
\left\|\Sigma_{O G K}\right\| \leq\left\|D^{-1}\right\|^{2}\|\Gamma\| .
$$

From (7), using [9, Th. 5.6.9], valid for any matrix norm, the unitary property of $E$, and Schwarz inequality

$$
\begin{aligned}
\sigma\left(C_{Z_{x x}}\right)^{2} & \leq\left\|C_{Z}\right\| \leq\left\|E^{\mathrm{T}}\right\|\left\|D^{-1}\right\|\left\|C_{X}\right\|\left\|D^{-1}\right\|\|E\| \\
& \leq\left\|D^{-1}\right\|^{2}\left\|C_{X}\right\|
\end{aligned}
$$

(a similar expression can be written for $\sigma\left(C_{Z_{y y}}\right)^{2}$ ). Substituting in (8)

$$
\left\|\Sigma_{\mathrm{OGK}}\right\|_{2} \leq\left\|D^{-1}\right\|_{2}^{4}\left\|C_{X}\right\|_{2} .
$$

Using the explicit calculation for the eigenvalues of $C_{X}$, it can be easily verified that

$$
\begin{aligned}
\|D\|_{2} & =\max \left\{\sigma\left(C_{X_{x x}}\right)^{2}, \sigma\left(C_{X_{y y}}\right)^{2}\right\}=\lambda_{1}(D) \leq \lambda_{1}\left(C_{X}\right) \\
\left\|D^{-1}\right\|_{2} & =\max \left\{\frac{1}{\sigma\left(C_{X_{x x}}\right)^{2}}, \frac{1}{\sigma\left(C_{X_{y y}}\right)^{2}}\right\}=\lambda_{1}\left(D^{-1}\right)=\frac{1}{\lambda_{2}(D)}
\end{aligned}
$$

and since $\lambda_{2}\left(C_{X}\right) \leq \lambda_{2}(D)$

$$
\left\|D^{-1}\right\|_{2} \leq \frac{1}{\lambda_{2}\left(C_{X}\right)}
$$

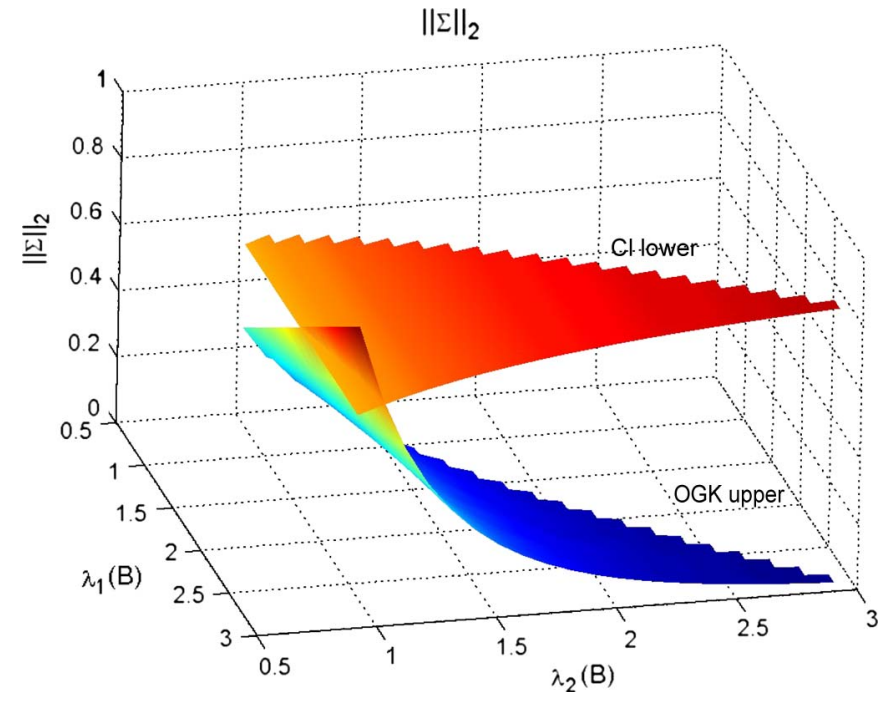

Fig. 8. OGK upper bound and CI lower bound.

Since $C_{X}$ is Hermitian, $\lambda_{1}\left(C_{X}\right)=\left\|C_{X}\right\|_{2}$, and the lower bound form for Weyl's inequalities (see [9, Th. 4.3.1]) is

$$
\left\|\Sigma_{O G K}\right\|_{2} \leq \frac{\lambda_{1}\left(C_{X}\right)}{\lambda_{2}\left(C_{X}\right)^{4}} \leq \frac{\lambda_{1}\left(A_{X}\right)+\lambda_{1}\left(B_{X}\right)}{\left(\lambda_{2}\left(A_{X}\right)+\lambda_{2}\left(B_{X}\right)\right)^{4}} .
$$

Fig. 8 shows the behavior of the upper bound (10) superimposed on the lower bound that was previously obtained for the CI procedure. Under the aforementioned assumption of equal means for the measurements by each of the data sources, it is clear that there are regions of the spectrum where each of the estimators outperforms the other. By selecting a different parameter for the OGK estimate, it is possible to increase the region in which the OGK outperforms (in the 2-norm sense) the $\mathrm{CI}$ and hence compensate for the deviations eventually when the aforementioned assumption does not hold.

\section{ACKNOWLEDGMENT}

J. Sequeira was working with the Autonomous Systems Group, Cranfield University, Shrivenham, U.K., during a sabbatical leave from the Instituto Superior Técnico, Universidade Técnica de Lisboa, under FCT Grant SFRH/BSAB/881/2009.

\section{REFERENCES}

[1] I. A. R. Ashokaraj, P. M. G. Silson, A. Tsourdos, and B. A. White, "Robust sensor-based navigation for mobile robots," IEEE Trans. Instrum. Meas., vol. 58, no. 3, pp. 551-556, Mar. 2009.

[2] N. A. Campbell, H. P. Lopuhaa, and P. J. Rousseeuw, "On the calculation of a robust $S$-estimator of a covariance matrix," Stat. Med., vol. 17, no. 23, pp. 2685-2695, Dec. 1998.

[3] M. De Cecco, L. Baglivo, and F. Angrilli, "Real-time uncertainty estimation of autonomous guided vehicle trajectory taking into account correlated and uncorrelated effects," IEEE Trans. Instrum. Meas., vol. 56 , no. 3, pp. 696-703, Jun. 2007.

[4] J. Chen, A. Bandoni, and J. A. Romagnoli, "Robust estimation of measurement error variance/covariance from process sampling data," Comput. Chem. Eng., vol. 21, no. 6, pp. 593-600, Feb. 1997.

[5] S. Copt and M. Victoria-Feser, Fast Algorithms for Computing High Breakdown Covariance Matrices With Missing Data, Aug. 2003. 
[6] D. Fränken and A. Hüpper, "Improved fast covariance intersection for distributed data fusion," in Proc. 8th Int. Conf. Inf. Fusion, Philadelphia, PA, Jul. 2005, pp. 154-160.

[7] W. Fulton, "Eigenvalues of sums of Hermitian matrices," in Proc. 50th Sémin. BOURBAKI 1997-1998, Jun. 1998, vol. 845, pp. 255-269.

[8] R. Gnanadesikan and J. R. Kettenring, "Robust estimates, residuals, and outlier detection with multiresponse data," Biometrics, vol. 28, no. 1, pp. 81-124, Mar. 1972.

[9] R. A. Horn and C. R. Johnson, Matrix Analysis, 21st ed. Cambridge, U.K.: Cambridge Univ. Press, 2007.

[10] A. Jazwinski, Stochastic Processes and Filtering Theory. New York: Academic, 1970.

[11] S. J. Julier and J. K. Uhlmann, "A non-divergent estimation algorithm in the presence of unknown correlations," in Proc. Amer. Control Conf., Albuquerque, NM, Jun. 1997, pp. 2369-2373.

[12] S. J. Julier and J. K. Uhlmann, "Using covariance intersection for SLAM," J. Robot. Autonom. Syst., vol. 55, no. 1, pp. 3-20, Jan. 2007.

[13] M. Montemerlo, S. Thrun, D. Koller, and B. Wegbreit, "FastSLAM: A factored solution to the simultaneous localization and mapping problem," in Proc. 8th AAAI Nat. Conf. Artif. Intell., Edmonton, AB, Canada, 2002, pp. 593-598.

[14] A. I. Mourikis and S. I. Roumeliotis, "Predicting the performance of cooperative simultaneous localization and mapping (C-SLAM)," Int. $J$. Robot. Res., vol. 25, no. 12, pp. 1273-1286, Dec. 2006.

[15] N. Wang and A. E. Raftery, "Nearest-neighbor variance estimation (NNVE): Robust covariance estimation via nearest-neighbor cleaning," J. Amer. Stat. Assoc., vol. 97, no. 460, pp. 994-1019, Dec. 2002.

[16] A. Papoulis, Probability, Random Variables and Stochastic Processes, 2nd ed. New York: McGraw-Hill, 1984.

[17] J. Sequeira, A. Tsourdos, and S. Lazarus, "Robust covariance estimation in sensor data fusion," in Proc. IEEE Int. Workshop SSRR, Denver, CO, Nov. 3-6, 2009, pp. 1-7.

[18] C.-C. Tsai, "A localization system of a mobile robot by fusing deadreckoning and ultrasonic measurements," IEEE Trans. Instrum. Meas., vol. 47, no. 5, pp. 1399-1404, Oct. 1998.

[19] J. K. Uhlmann, "Covariance consistency methods for fault-tolerant distributed data fusion," J. Inf. Fusion, vol. 4, no. 3, pp. 201-215, Sep. 2003.

[20] P. Vadakkepat and L. Jing, "Improved particle filter in sensor fusion for tracking randomly moving object," IEEE Trans. Instrum. Meas., vol. 55, no. 5, pp. 1823-1832, Oct. 2006.

[21] M. Walter and J. Leonard, "An experimental investigation of cooperative SLAM," in Proc. 5th IFAC Symp. IAV, Lisbon, Portugal, Jul. 2004.

[22] T. Yang and V. Aitken, "Evidential mapping for mobile robots with range sensors," IEEE Trans. Instrum. Meas., vol. 55, no. 4, pp. 1422-1429, Aug. 2006.

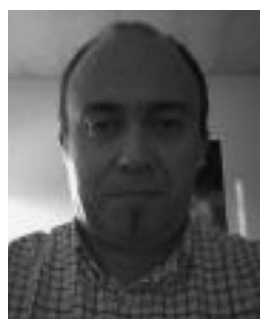

João Sequeira received the Ph.D. degree in cooperative robotics from the Instituto Superior Técnico (IST), Universidade Técnica de Lisboa, Lisbon, Portugal, in 1999.

He is currently an Assistant Professor with IST, Universidade Técnica de Lisboa, where he is a Senior Researcher in the Institute for Systems and Robotics. He participated in several national and international research projects, has been in program committees for several international conferences, and acts regularly as a Reviewer for various international journals and conferences.

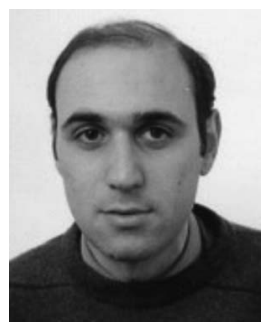

Antonios Tsourdos (S'97-M'99) received the Ph.D degree in robust nonlinear control flight control systems design from Cranfield University, Cranfield, U.K.

$\mathrm{He}$ is currently a Professor and the Head of the Autonomous Systems Group, Department of Informatics and Systems Engineering, Cranfield University at the U.K. Defence Academy, Shrivenham, U.K. He is an Editorial Board Member for the International Journal of Systems Science and the Proceedings of the Institution of Mechanical Engineers-Part G: Journal of Aerospace Engineering. He has been a Reviewer for several professional journals and a Committee Member for various international conferences.

Prof. Tsourdos is a member of the American Institute of Aeronautics and Astronautics Technical Committee on Guidance, Control, and Navigation and the International Federation of Automatic Control Technical Committee on Aerospace Control. He is also an Editorial Board Member for the IEEE TRANSACTIONS ON InStRUMENTATION AND MEASUREMENT.

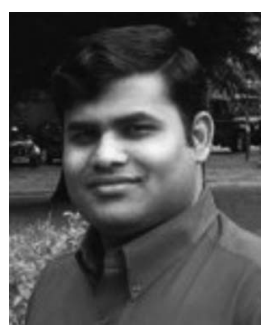

Samuel B. Lazarus received the Ph.D. degree in sensor-based navigation from Cranfield University, Cranfield, U.K., in 2009.

$\mathrm{He}$ is currently a Postdoctoral Researcher with the Autonomous Systems Group, Department of Informatics and Systems Engineering, Cranfield University at the U.K. Defence Academy, Shrivenham, U.K. His research interests include sensor fusion, sensorbased navigation, and guidance for both aerial and land vehicles. 